\title{
Cuidado realizado por enfermeiro não especialista em oncologia ao cliente oncológico hospitalizado: Revisão integrativa
}

\author{
Care provided by a non-specialist oncology nurse to hospitalized cancer patients: An integrative
}

Review

Atención brindada por una enfermera oncológica no especializada a pacientes oncológicos

hospitalizados: Una revisión integradora

Recebido: 21/04/2021 | Revisado: 26/04/2021 | Aceito: 05/05/2021 | Publicado: 19/05/2021

Dasymar Martins da Silva Lucas

ORCID: https://orcid.org/0000-0002-6380-6031

Universidade Federal do Estado do Rio de Janeiro, Brasil

E-mail: dasy_lucas@hotmail.com

Sônia Regina de Souza

ORCID: https://orcid.org/0000-0001-7981-0038

Universidade Federal do Estado do Rio de Janeiro, Brasil

E-mail: soniasilvio@gmail.com

Jenniffer Lopes Rodrigues da Silva

ORCID: https://orcid.org/0000-0003-4117-6697

Universidade Federal do Estado do Rio de Janeiro, Brasil

E-mail: jenny.junior13@gmail.com

Laisa Figueiredo Ferreira Lós de Alcantara

ORCID: https://orcid.org/0000-0002-2435-7616

Universidade Federal do Estado do Rio de Janeiro, Brasil

E-mail: dra.laisa@gmail.com

Eliza Cristina Macedo

ORCID: https://orcid.org/0000-0001-8824-9107

Universidade Federal do Estado do Rio de Janeiro, Brasil

E-mail: macedo.unirio@gmail.com

Adriana da Costa Coelho

ORCID: https://orcid.org/0000-0003-2047-9592

Universidade Federal do Estado do Rio de Janeiro, Brasil

E-mail: dricoelho10@hotmail.com

Maria Clara Mellis Rodrigues

ORCID: https://orcid.org/0000-0002-5941-3171 Universidade Federal do Estado do Rio de Janeiro, Brasil

E-mail: mariaclaramellis@gmail.com

Maria Odete Queiroz Lima Tavares

ORCID: https://orcid.org/0000-0003-4399-1409

Universidade Federal do Estado do Rio de Janeiro, Brasil

E-mail: odetequeiroz34@gmail.com

\begin{abstract}
Resumo
O câncer se caracteriza pelo crescimento descontrolado e disseminação de células anormais no organismo, cujas causas podem estar associadas a fatores externos ou internos em crescente incidência. Com isso, os profissionais de saúde ainda pecam aos cuidados devido à falta de conhecimento no manejo da doença. Objetivo: identificar as evidências científicas disponíveis sobre o cuidado realizado por enfermeiro não especialista em oncologia ao cliente oncológico hospitalizado. Metodologia: Trata-se de uma revisão integrativa, utilizando quatro bases de dados: Pubmed, Scielo, Cinahl e Lilacs para a seleção de estudos em português, inglês e espanhol, dos anos de 2008 a 2018. Resultados: Constitui uma amostra final de nove artigos. Após análise, emergiram as seguintes categorias: intervenções no tratamento quimioterápico; cuidados com feridas tumorais; conforto ao paciente/família; atendimento às necessidades específicas do cliente; busca de qualidade de vida ao portador de doença oncológica; apoio espiritual/ religioso, emocional; elaboração do cuidado e subconjunto terminológico para melhorias do processo de enfermagem; busca pela minimização da dor relacionada às feridas tumorais; cuidados paliativos, prevenção do sofrimento e ressignificação da gerência do cuidado. Conclusão: Enfermeiros não especialistas realizam diversos cuidados oncológicos. Entretanto, desconhecem seu protagonismo como agente de mudanças, caracterizando lacunas nessa temática. Esta pesquisa é relevante para a Enfermagem, por minimizar dicotomias entre teoria/prática e qualidade de vida dos clientes. Destaca-se a Educação Permanente como ferramenta potente para qualificação e otimização do cuidado. Ressalta-se a necessidade de mais pesquisas e estudos.
\end{abstract}


Palavras-chave: Enfermeiro; Oncologia; Cuidados de enfermagem; Pacientes Internados.

\begin{abstract}
Cancer is characterized by uncontrolled growth and the spread of abnormal cells in the body, the causes of which may be associated with external or internal factors with increasing incidence. Thus, health professionals still sin in care due to the lack of knowledge in the management of the disease. Objective: To identify the available scientific evidence on the care provided by a non-specialist oncology nurse to hospitalized cancer patients. Methodology: This is an integrative review, using four databases: Pubmed, Scielo, Cinahl, and Lilacs for the selection of studies in Portuguese, English and Spanish, from the years 2008 to 2018. Results: It constitutes a final sample of nine articles. After analysis, the following categories emerged: interventions in chemotherapy treatment; care for tumor wounds; comfort to the patient/family; meeting the specific needs of the client; search for quality of life for cancer patients; spiritual/religious, emotional support; elaboration of care and terminological subset to improve the nursing process; seeking to minimize pain related to tumor wounds; palliative care, prevention of suffering and reframing of care management. Conclusion: Non-specialist nurses perform various cancer care. However, they are unaware of their role as agents of change, characterizing gaps in this theme. This research is relevant for Nursing, as it minimizes dichotomies between theory/practice and clients' quality of life. Permanent Education stands out as a powerful tool for qualifying and optimizing care. The need for further research and studies is emphasized.
\end{abstract}

Keywords: Nurse; Oncology; Nursing care; Inpatients.

\title{
Resumen
}

El cáncer se caracteriza por un crecimiento descontrolado y la diseminación de células anormales en el cuerpo, cuyas causas pueden estar asociadas con factores externos o internos con una incidencia creciente. Así, los profesionales de la salud siguen pecando en la atención por el desconocimiento en el manejo de la enfermedad. Objetivo: Identificar la evidencia científica disponible sobre la atención brindada por enfermero oncológico no especialista en pacientes oncológicos hospitalizados. Metodología: Se trata de una revisión integradora, utilizando cuatro bases de datos: Pubmed, Scielo, Cinahl y Lilacs para la selección de estudios en portugués, inglés y español, de los años 2008 a 2018. Resultados: Constituye una muestra final de nueve artículos. Después del análisis, surgieron las siguientes categorías: intervenciones en el tratamiento de quimioterapia; cuidado de heridas tumorales; comodidad para el paciente / familia; satisfacer las necesidades específicas del cliente; búsqueda de calidad de vida para pacientes con cáncer; apoyo espiritual / religioso, emocional; elaboración de cuidados y subconjunto terminológico para mejorar el proceso de enfermería; buscando minimizar el dolor relacionado con las heridas del tumor; cuidados paliativos, prevención del sufrimiento y replanteamiento de la gestión asistencial. Conclusión: enfermeras no especializadas realizan diversos cuidados oncológicos. Sin embargo, desconocen su rol como agentes de cambio, lo que caracteriza brechas en este tema. Esta investigación es relevante para Enfermería, ya que minimiza las dicotomías entre teoría / práctica y la calidad de vida de los clientes. La Educación Permanente se destaca como una poderosa herramienta para calificar y optimizar la atención. Se enfatiza la necesidad de realizar más investigaciones y estudios.

Palabras clave: Enfermera; Oncología, Atención de enfermería; Pacientes hospitalizados.

\section{Introdução}

O câncer se caracteriza pelo crescimento descontrolado e disseminação de células anormais no organismo, cujas causas podem estar associadas a fatores externos, como o estilo de vida e o excesso de peso corporal, e/ou fatores internos não modificáveis, como as mutações genéticas hereditárias, hormônios, e condições imunológicas (Instituto Nacional de Câncer José Alencar Gomes da Silva [INCA], 2020).

Conforme detectado pelo INCA (2020), ocorreram no ano passado 309.750 novos casos de câncer em homens e 316.280 em mulheres no Brasil, estimando-se para cada ano do triênio 2020-2022, 625 mil casos novos.

A tendência de aumento dos casos está diretamente relacionada à maior exposição aos fatores de risco cancerígenos, aos atuais padrões de vida adotados e resultantes de um processo de industrialização cada vez mais avançado (Lins \& Souza, 2018).

A patologia é considerada um problema de saúde pública, devido a sua incidência, custos gerados como consequência dos tratamentos mais caros e prolongados, assim como os custos previdenciários consequentes do afastamento do trabalho, somatizados às mortes prematuras, anos de incapacidade que diminuem a contribuição social do indivíduo para o desenvolvimento da nação e da sua família (Paiva \& Cesse, 2015). 
Políticas públicas de saúde surgiram ao longo dos anos objetivando o combate ao aumento dos casos e estruturando ações à atenção oncológica envolvendo as três esferas do governo para uma melhor assistência. A atuação da enfermagem diante desta assistência oncológica visa um atendimento integral, desde a avaliação diagnóstica, do tratamento até a reabilitação. Sendo o paciente oncológico muitas vezes portador de dor crônica, cabe ao enfermeiro avaliar a dor do paciente, considerando-a como um sinal vital que pode ser mensurado por meio de escalas, e não apenas relacionada aos aspectos subjetivos, para que somente assim, seja capaz de criar estratégias eficazes para o controle da mesma (Rolim et al., 2019). Além do cuidado físico, o apoio psicossocial, conforto emocional e espiritual, com uma comunicação clara e consistente, fazse necessária na atuação da assistência constantemente (Silva et al.,2020).

Neste contexto, cogita-se o escopo da formação profissional voltado à prevenção, promoção, proteção e reabilitação da saúde, como expresso no currículo generalista da formação do Enfermeiro. No entanto, quando se trata dos cuidados aos pacientes oncológicos, a sensação de inaptidão para esse cuidado específico irrompe gerando a necessidade de contínuo aprimoramento dos conhecimentos técnico-científicos (Barp et al., 2018).

Este acontecimento ocorre devido ao perfil da formação de profissionais generalistas na graduação.A disciplina como a oncologia é vista como um ensino em nível específico e seu aprofundamento fomenta-se nas especializações indo de contramão ao contexto epidemiológico. Apesar do crescente número de especializações na enfermagem, dentro da assistência ao paciente com câncer, encontramos o enfermeiro não especialista (Frota et al., 2019).

Deste modo, o presente estudo justifica-se pela colaboração na qualificação da assistência considerando que a identificação dos cuidados realizados pelo enfermeiro ao paciente oncológico, bem como suas dificuldades, podem contribuir para alinhar ações de educação permanente, que visem à melhoria deste processo.

A partir do exposto, toma-se como objetivo desta pesquisa identificar o cuidado prestado por enfermeiro não especialista ao cliente oncológico hospitalizado.

\section{Metodologia}

\section{Desenho do estudo}

Foi realizada busca análise da literatura, através de levantamento bibliográfico, caracterizada como um método de pesquisa que utiliza a prática baseada em evidências. A Revisão Integrativa tem sido considerada como indispensável no campo da saúde, pois sintetiza pesquisas disponíveis sobre um assunto e permite o direcionamento da prática, fundamentandose em conhecimento científico (Gadelha, 2018).

Utilizou-se todas as etapas recomendadas, ou seja: estabelecimento de questão norteadora, definição de amostragem, categorização e avaliação de cada estudo, interpretação dos resultados e síntese das evidências advindas dos estudos (Polit \& Beck, 2018)

Para a elaboração da questão norteadora utilizou-se a estratégia PICo, proposta pelo Instituto Joanne Brigs (Mendes, Silveira \& Galvão, 2008).

Como apresentado na Tabela 1. Trata-se de um acrônimo onde o P corresponde aos participantes, I ao fenômeno de interesse e Co ao contexto do estudo (Cardoso et al., 2019).

Os termos controlados utilizados foram os descritores: nursing care; oncology; inpatients. O caractere "curinga" asterisco $(*)$ ou cifrão $(\$)$ trunca o final de uma palavra, ampliando os resultados para todos os sufixos que aparecem à partir do radical truncado. Deste modo, os termos nurse, nurses e nursing foram recuperados. 
Tabela 1. Descrição da estratégia PICo.

\begin{tabular}{llll}
\hline Acrônimo $=>$ & \multicolumn{1}{c}{ P } & I & Co \\
\hline Definição $=>$ & População & Fenômeno de Interesse & Contexto \\
\hline Descrição $=>$ & Enfermeiro não especialista em oncologia & Cuidado ao cliente oncológico & Hospitalização \\
\hline $\begin{array}{l}\text { Questão } \\
\text { elaborada }=>\end{array}$ & $\begin{array}{l}\text { Qual(is) cuidado(s) o enfermeiro não especialista em oncologia realiza ao cliente oncológico } \\
\text { hospitalizado? }\end{array}$ & \\
\hline
\end{tabular}

Fonte: Autores (2018).

A busca pelos estudos de interesse foi realizada através da Biblioteca Virtual em Saúde (BVS), desenvolvida sob coordenação do Centro Latino-americano de Informação em Ciências da Saúde (BIREME) nas seguintes coleções eletrônicas: Medline, LILACS e BDENF. E ainda na base Cumulative Index to Nursing and Allied Health Literature com as seguintes estratégias na Tabela 2, recuperadas do histórico de buscas das respectivas bases:

Tabela 2. Descrição da estratégia de busca.

\begin{tabular}{l|l}
\hline \multicolumn{1}{c|}{ Medline LILACS BDENF } & \multicolumn{1}{c}{ CINAHL } \\
\hline tw: (nurs* AND neoplasms) AND & Nursing care AND (neoplasms or oncology or cancer) NOT oncology \\
(instance:"regional") AND ( full text: ("1") & nursing. Limiters - Full Text; Published Date: 2008-2018. Narrow by \\
AND mj: ("Enfermeiras e Enfermeiros") AND & Subject: Major - nursing knowledge; health behavior, quality of nursing \\
limit: ("adult") AND jd: ("Enfermagem"). & care, nursing practice, nurse attitudes, quality of life, oncologic care, \\
& nursing care. Language - Spanish, portuguese, english. Age - all adult. \\
\hline
\end{tabular}

Fonte: Autores (2018).

\section{População e cenário}

Enfermeiros não especialistas que prestam cuidados ao cliente oncológico hospitalizado. Nos estudos encontrados, buscou-se a correspondência com a descrição das competências básicas para Enfermeiros Oncológicos Clínicos Especialista (OCNSs - oncology clinical nurse specialists) pela Oncology Nursing Society (ONS) (2008), que foi tomada como referência central, considerando-se que as competências descritas enfatizam uma filosofia única de prática para a especialidade, dado às necessidades únicas de pacientes com um diagnóstico pregresso, atual ou potencial de câncer.

\section{Critérios de seleção}

Os critérios de inclusão foram estudos disponíveis na íntegra, em português, inglês e espanhol, publicados entre 2008 e 2018. O corte temporal inicial deve-se à descrição das competências básicas para Enfermeiros Oncológicos Clínicos Especialista (OCNSs - oncology clinical nurse specialists) pela ONS em 2008.

Excluíram-se textos duplicados, que fizessem clara referência a cuidados de enfermagem realizados por enfermeiros especialistas em oncologia, cuidados realizados pela equipe de enfermagem e/ou pela equipe multiprofissional.

\section{Definição da amostra}

Foram identificados 59 estudos na CINAHL, 32 na Pubmed, três na LILACS e nenhum na BDENF, perfazendo o total de 94 artigos pré- selecionados.

Após a leitura criteriosa dos títulos, resumos e descritores dos 94 artigos encontrados, 34 estudos foram elegidos para serem lidos na íntegra e destes, nove compuseram a amostra final. O Fluxograma apresentado na Figura 1 descreve essa etapa: 
Figura 1. Seleção de artigos: Fluxograma.

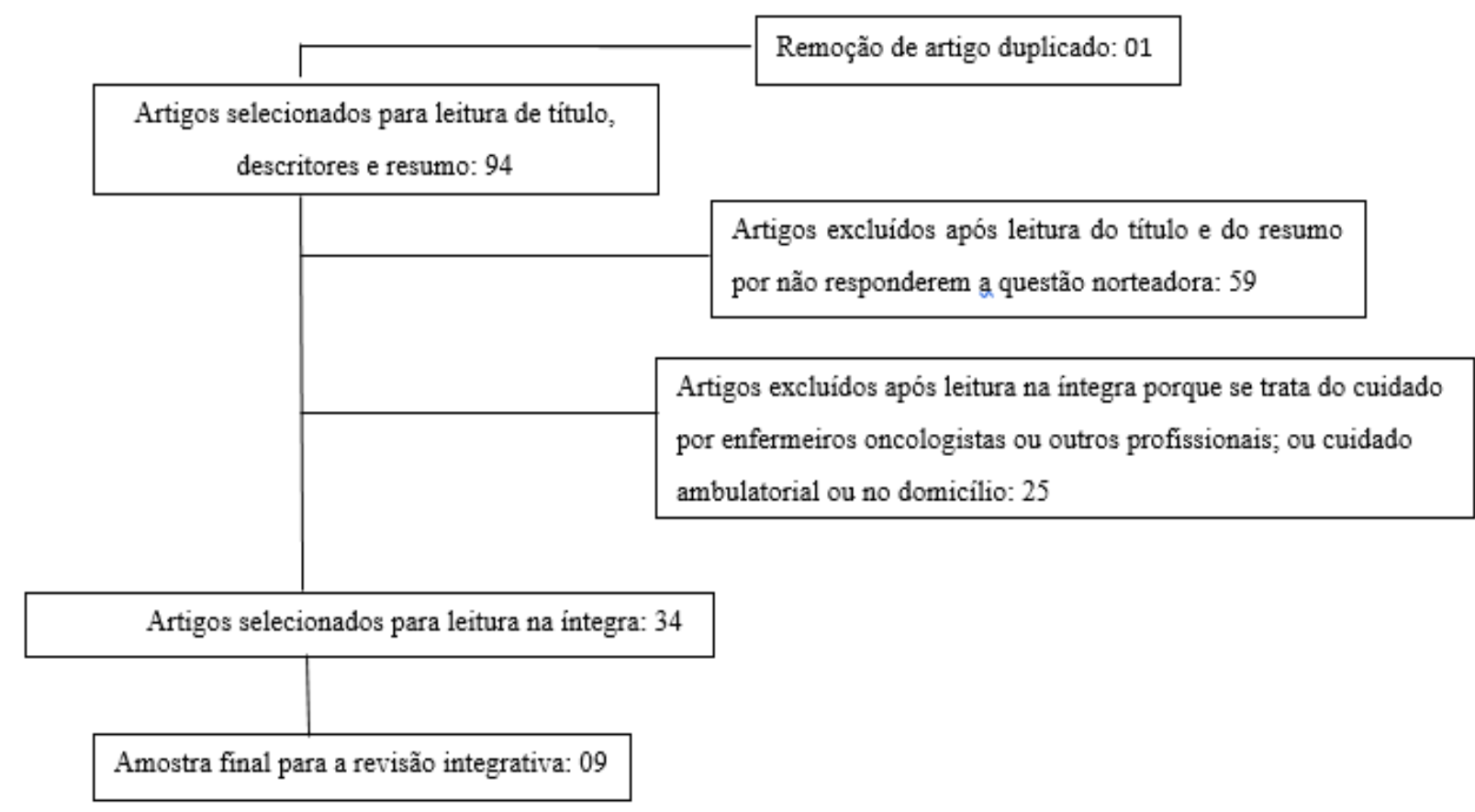

Fonte: Autores (2018).

O levantamento de dados foi realizado a partir da leitura minuciosa das competências básicas para Enfermeiros Oncológicos Clínicos Especialista associada aos estudos encontrados por meio de análise de conteúdo e ressaltando que se buscou essa correspondência, a fim de melhor organizar a apresentação dos resultados encontrados a partir de um referencial de enfermagem.

Como estratégia para extrair as informações necessárias dos estudos da amostra final, elaborou-se um meio de viabilizar visualmente a análise de cada artigo de maneira resumida, apresentando suas diferenças de acordo com variáveis determinadas (Ursi, 2005). Para tal, desenvolveu-se um instrumento de coleta de dados composto por: título; periódico/data/local; autor; desenho de estudo; nível de evidência e qualidade metodológica.

Considerando o delineamento dos níveis de evidência dos estudos, utilizou-se uma classificação baseada em Stillwell et al. (2010), em Revisão sistemática ou metanálise I, Estudo Randomizado Controlado II, Estudo Controlado com Randomização III, Estudo Caso-controle ou Estudo de Coorte IV, Revisão Sistemática de Estudos Qualitativos ou Descritivos V, Estudo qualitativo ou descritivo VI, Opinião ou Consenso VII.

Ao analisar os nove artigos incluídos na presente revisão, observou-se que foram publicados a partir de 2014 (A6) até o ano 2018 (A1); sendo a maioria publicada em 2015 (A2, A4 e A8). Em relação ao desenho de estudo, ao nível de evidência e avaliação da qualidade segue a apresentação conforme tabela 3 a seguir: 
Research, Society and Development, v. 10, n. 6, e2910615306, 2021

(CC BY 4.0) | ISSN 2525-3409 | DOI: http://dx.doi.org/10.33448/rsd-v10i6.15306

Tabela 3. Perfil dos artigos analisados.

\begin{tabular}{|c|c|c|c|c|c|}
\hline Artigo & Título & $\begin{array}{l}\text { Periódico/D } \\
\text { ata }\end{array}$ & Autor & $\begin{array}{l}\text { Desenho de } \\
\text { estudo }\end{array}$ & $\begin{array}{l}\text { Nível de } \\
\text { evidência }\end{array}$ \\
\hline A1 & $\begin{array}{l}\text { Orientações de } \\
\text { cuidado do } \\
\text { enfermeiro para a } \\
\text { mulher em tratamento } \\
\text { para câncer de mama }\end{array}$ & $\begin{array}{l}\text { Nurse UFPE } \\
\text { on line, } 12 \\
\text { (3) } 2018 .\end{array}$ & $\begin{array}{l}\text { Ferrari, C.F., } \\
\text { et al. }\end{array}$ & $\begin{array}{l}\text { Estudo } \\
\text { qualitativo e } \\
\text { descritivo. }\end{array}$ & VI \\
\hline A2 & $\begin{array}{l}\text { Cuidados paliativos } \\
\text { de enfermagem a } \\
\text { paciente com } \\
\text { carcinoma } \\
\text { Basocelular } \\
\text { Terebrante: estudo de } \\
\text { caso }\end{array}$ & $\begin{array}{l}\text { Rev enferm } \\
\text { UFPE on } \\
\text { line, } 9 \text { (11) } \\
2015 .\end{array}$ & $\begin{array}{l}\text { Agra, G., et } \\
\text { al. }\end{array}$ & Estudo de caso. & VII \\
\hline A3 & $\begin{array}{l}\text { Cuidados de } \\
\text { enfermagem a } \\
\text { pacientes onco- } \\
\text { hematológicos } \\
\text { submetidos a altas } \\
\text { doses de } \\
\text { quimioterapia: revisão } \\
\text { integrativa }\end{array}$ & $\begin{array}{l}\text { J Nurs } \\
\text { UFPE on } \\
\text { line, } 10 \\
\text { (Suppl. } \\
\text { 3): } 1544-55 \text {, } \\
2016 .\end{array}$ & $\begin{array}{l}\text { Carlucci } \\
\text {,V.D.S., et al. }\end{array}$ & $\begin{array}{l}\text { Revisão } \\
\text { integrativa }\end{array}$ & $\mathrm{V}$ \\
\hline A4 & $\begin{array}{l}\text { Gerência do cuidado } \\
\text { de enfermagem ao } \\
\text { homem com câncer }\end{array}$ & $\begin{array}{l}\text { Rev. Pesqui. } \\
\text { Cuid. } \\
\text { Fundam. } \\
\text { (Online), } 7 \\
(3), \\
2015 .\end{array}$ & $\begin{array}{l}\text { Mesquita, M. } \\
\text { G. R., et al. }\end{array}$ & Qualitativo & VI \\
\hline A5 & $\begin{array}{l}\text { Intervenções de } \\
\text { enfermagem para } \\
\text { pacientes oncológicos } \\
\text { com odor fétido em } \\
\text { ferida tumoral }\end{array}$ & $\begin{array}{l}\text { Aquichán.17 } \\
\text { (3), } \\
2017 .\end{array}$ & $\begin{array}{l}\text { Freitas, C., et } \\
\text { al. }\end{array}$ & $\begin{array}{l}\text { Revisão } \\
\text { integrativa }\end{array}$ & $\mathrm{V}$ \\
\hline A6 & $\begin{array}{l}\text { A atuação do } \\
\text { enfermeiro junto à } \\
\text { criança com câncer: } \\
\text { cuidados paliativos }\end{array}$ & $\begin{array}{l}\text { Rev. enferm. } \\
\text { UERJ, } 22 \\
(6), \\
2014 .\end{array}$ & $\begin{array}{l}\text { Monteiro, A. } \\
\text { C. M., et al. }\end{array}$ & $\begin{array}{l}\text { Qualitativo } \\
\text { descritivo }\end{array}$ & VI \\
\hline A7 & $\begin{array}{l}\text { A dimensão espiritual } \\
\text { no processo de cuidar } \\
\text { da pessoa com câncer: } \\
\text { uma revisão } \\
\text { integrativa }\end{array}$ & $\begin{array}{l}\text { Cultura de } \\
\text { los Cuidados } \\
\text { (Edición } \\
\text { digital), } 21 \\
\text { (47). }\end{array}$ & $\begin{array}{l}\text { Simão- } \\
\text { Miranda, T.P., } \\
\text { et al. }\end{array}$ & $\begin{array}{l}\text { Revisão } \\
\text { integrativa }\end{array}$ & $\mathrm{V}$ \\
\hline A8 & $\begin{array}{l}\text { Percepções de } \\
\text { enfermeiros e manejo } \\
\text { da dor de pacientes } \\
\text { oncológicos }\end{array}$ & $\begin{array}{l}\text { REME rev. } \\
\text { min. enferm, } \\
19(3), \\
2015 .\end{array}$ & $\begin{array}{l}\text { Stübe, M., et } \\
\text { al. }\end{array}$ & $\begin{array}{l}\text { Qualitativo } \\
\text { descritivo }\end{array}$ & VI \\
\hline A9 & $\begin{array}{l}\text { Providing Palliative } \\
\text { Care for Cancer } \\
\text { Patients: Meeting the } \\
\text { Needs in Kenya. }\end{array}$ & $\begin{array}{l}\text { Ann \& } \\
\text { Joshua } \\
\text { Medical } \\
\text { Publishing } \\
\text { Co, } \\
2017\end{array}$ & $\begin{array}{l}\text { Malloy, P., et } \\
\text { al }\end{array}$ & Estudo de caso & VII \\
\hline
\end{tabular}

Fonte: Autores (2018). 
Quanto à qualidade metodológica foi utilizada a lista de avaliação Critical Ap praisal Skills Program me (CASP), Public Health Resource Unit (PHRU) da Universidade de Oxford. A ferramenta possui dez itens, contabilizando o máximo de dez pontos, e classificando os estudos com as seguintes pontuações: 6 a 10 pontos com boa qualidade metodológica e viés reduzido e o mínimo de 5 pontos com qualidade metodológica satisfatória com risco de viés aumentado.

\section{Análise e tratamento dos dados}

Os itens para serem analisados e avaliados em estudos qualitativos são:

1) objetivo claro e justificado;

2) metodologia adequada;

3) apresentação e discussão dos procedimentos metodológicos;

4) seleção adequada da amostra;

5) coleta de dados detalhada;

6) aspectos éticos;

7) análise de dados rigorosa e fundamentada;

8) apresentação e discussão dos resultados;

9) contribuições e indicações de novas questões de pesquisa.

\section{Aspectos éticos}

A Resolução $N^{\circ}$ 510, de 07 de abril de 2016 dispõe sobre as normas aplicáveis a pesquisas em Ciências Humanas e Sociais. Seguindo-a, respeitou-se os procedimentos metodológicos envolvidos na utilização de dados diretamente obtidos com os participantes ou de informações identificáveis ou que possam acarretar riscos maiores do que os existentes na vida cotidiana. De maneira que a pesquisa realizada foi desenvolvida exclusivamente com textos científicos para revisão da literatura científica sem a necessidade de ser registrada nem avaliada pelo sistema CEP/CONEP (Conselho Nacional de Saúde [CNS], 2016).

\section{Resultados}

Todos os artigos incluídos neste estudo tiveram enfoque no cuidado realizado por enfermeiro não especialista em oncologia ao cliente oncológico hospitalizado, entretanto, nenhum dos autores descreveu desta forma a assistência prestada.

Os cuidados identificados na revisão foram divididos em três categorias conforme a correspondência encontrada com a descrição das competências básicas dos OCNS tomada como referência central, enfatizando as competências como filosofia única de melhores práticas junto ao cliente oncológico de acordo com a ONS em algumas esferas: Esfera de influência paciente/cliente, Esfera de influência do enfermeiro e da prática de enfermagem e Esfera da organização dos sistemas (ONS,2008).

Segue no quadro abaixo a correspondência realizada entre os artigos utilizados no estudo, considerando-se seus principais resultados e as competências descritas acima: 
Tabela 4. Correspondência entre cuidados de Enfermeiros não especialistas em oncologia e as competências básicas de Enfermeiros Oncológicos Clínicos Especialista (OCNSs).

\begin{tabular}{|c|c|c|}
\hline Artigos & $\begin{array}{l}\text { Cuidados realizados pelo o enfermeiro não especialista em oncologia ao } \\
\text { cliente oncológico hospitalizado: principais resultados e conclusões. }\end{array}$ & Competências básicas \\
\hline A1 - Ferrari, C.F., et al. & $\begin{array}{l}\text { O estudo enfatiza que apesar dos avanços na área do tratamento } \\
\text { quimioterápico, ainda há muito a alcançar dentre riscos e benefícios para o } \\
\text { paciente. Relata que o Enfermeiro presta cuidado de forma direta à mulher com } \\
\text { câncer sendo de grande importância para que a paciente consiga enfrentar-lo da } \\
\text { melhor maneira. O estudo observou que enfermeiros realizam orientações e } \\
\text { intervenções que pontuais, embasadas na teoria, de extrema relevância frente } \\
\text { às queixas relacionadas aos efeitos colaterais do tratamento quimioterápico } \\
\text { relatadas pelas pacientes, salientando o protagonismo desse profissional no } \\
\text { cuidado e na supervisão do mesmo. }\end{array}$ & $\begin{array}{l}\text { I - Esfera Influência do } \\
\text { Enfermeiro e da prática de } \\
\text { enfermagem }\end{array}$ \\
\hline A2 - Agra, G; et al. & $\begin{array}{l}\text { No estudo Enfermeiros promoveram conforto ao paciente durante a internação } \\
\text { hospitalar e domiciliar, sendo primordiais para controle dos sinais e sintomas } \\
\text { de lesões tumorais, promovendo dignidade durante o processo de } \\
\text { terminalidade. Informa que pela complexidade, o carcinoma apresentado pelo } \\
\text { paciente, constitui um desafio para os enfermeiros que estes compõem a equipe } \\
\text { multiprofissional de cuidados paliativos e são responsáveis pela avaliação do } \\
\text { paciente, da lesão, escolha da cobertura e de curativos e necessita desenvolver } \\
\text { competências e habilidades a fim de identificar, avaliar e tratar estas lesões, } \\
\text { proporcionando assistência integral ao paciente e sua família. }\end{array}$ & $\begin{array}{l}\text { I - Esfera Influência do } \\
\text { Enfermeiro e da prática de } \\
\text { enfermagem }\end{array}$ \\
\hline $\begin{array}{l}\text { A3-Carlucci, V.D.S, et } \\
\text { al. }\end{array}$ & $\begin{array}{l}\text { Nos estudos as intervenções e cuidado ao paciente submetido a altas doses de } \\
\text { quimioterapia seguidas ou não ao Transplante de Células-Tronco } \\
\text { Hematopoéticas foram, em sua maioria, para minimizar os efeitos adversos do } \\
\text { tratamento e se relacionaram ao prejuízo funcional, nutricional, na qualidade } \\
\text { do sono, repouso e qualidade de vida. Portanto, apresentou que cabe ao } \\
\text { enfermeiro as estratégias para controle da situação do paciente para evitar } \\
\text { complicações, minimizando ou promovendo melhoras desse quadro. }\end{array}$ & $\begin{array}{l}\text { I - Esfera Influência do } \\
\text { Enfermeiro e da prática de } \\
\text { enfermagem }\end{array}$ \\
\hline $\begin{array}{l}\text { A4 - Mesquita, M. G. R., } \\
\text { et al. }\end{array}$ & $\begin{array}{l}\text { Cinco categorias emergiram no estudo gerando o fenômeno: Ressignificando a } \\
\text { gerência do cuidado de enfermagem mediante a perspectiva de gênero, a fim de } \\
\text { assegurar a integralidade ao homem com câncer. O estudo mostrou que na } \\
\text { interação com homens em tratamento antineoplásico, os enfermeiros respeitam } \\
\text { a influência da masculinidade em suas vidas e detectam a necessidade de } \\
\text { inclusão da perspectiva de gênero na prática profissional. }\end{array}$ & $\begin{array}{l}\text { II - Esfera de influência } \\
\text { paciente/cliente }\end{array}$ \\
\hline A5 - Freitas, C. M. et al. & $\begin{array}{l}\text { O estudo diversas práticas são executadas em pacientes com câncer avançado, } \\
\text { destacando-se atendimento de feridas tumorais, que acometem a pele e } \\
\text { desfiguram o corpo, tornando-se friáveis, dolorosas, com sinais clínicos e } \\
\text { sintomas limitantes, na maioria dos casos, nos últimos seis meses de vida. Com } \\
\text { este estudo e elaboração do subconjunto terminológico, ressaltou-se a } \\
\text { importância do enfermeiro diante do cuidado baseado em evidências } \\
\text { científicas, que reverta em segurança e subsídios para uma ação de } \\
\text { enfermagem segura no manejo dos sinais e dos sintomas das feridas tumorais e } \\
\text { também em dados para o ensino e para a pesquisa, levando a novos } \\
\text { conhecimentos para facilitar o processo gerencial e a tomada de decisão além } \\
\text { de fornecer base consistente na documentação da prática da enfermagem. }\end{array}$ & $\begin{array}{l}\text { II - Esfera de influência } \\
\text { paciente/cliente }\end{array}$ \\
\hline $\begin{array}{l}\text { A6 - Monteiro, A. C. M., } \\
\text { et al.. }\end{array}$ & $\begin{array}{l}\text { No estudo da atuação do enfermeiro junto à criança com câncer em cuidados } \\
\text { paliativos surgiram as categorias: Dar conforto à criança; Cuidar da família; } \\
\text { Atender às necessidades da criança; Proporcionar qualidade de vida à criança; } \\
\text { Dar apoio espiritual, emocional e religioso e Estar mais próximo da criança, } \\
\text { mostrando-se disponível o que possibilitou entender que, diante da criança com } \\
\text { câncer e família, o modo de agir do enfermeiro se pauta na promoção de } \\
\text { conforto e bem-estar, através do carinho e atenção, bem como o apoio } \\
\text { emocional e espiritual. }\end{array}$ & $\begin{array}{l}\text { I - Esfera Influência do } \\
\text { Enfermeiro e da prática de } \\
\text { enfermagem }\end{array}$ \\
\hline
\end{tabular}




\begin{tabular}{|c|c|c|}
\hline $\begin{array}{l}\text { A7 - Simão-Miranda, } \\
\text { T.P., et al. }\end{array}$ & $\begin{array}{l}\text { No estudo foi identificado que a equipe de enfermagem proporcionava alguns } \\
\text { cuidados espirituais ao paciente com câncer, dentre eles: meditação e a visita } \\
\text { de líder religioso. Concluiu-se que os enfermeiros desempenham atividades } \\
\text { que englobam cuidado espiritual a pessoa com câncer. Entretanto, há } \\
\text { necessidade que esse cuidado seja desempenhado por meio de embasamento } \\
\text { científico. }\end{array}$ & $\begin{array}{l}\text { I - Esfera Influência do } \\
\text { Enfermeiro e da prática de } \\
\text { enfermagem }\end{array}$ \\
\hline A8 - Stübe, M., et al. & $\begin{array}{l}\text { Os resultados encontrados na pesquisa resultaram em duas categorias } \\
\text { analíticas, uma relacionada à percepção de dor no paciente oncológico pela } \\
\text { enfermeira e a outra às ações direcionadas à redução da dor. Sinaliza mudanças } \\
\text { que podem e devem ser realizadas na minimização da dor e destaca: a } \\
\text { implantação da dor como quinto sinal vital, a formação de grupos de estudo e a } \\
\text { realização de pesquisas com enfoque na dor e assistência ao paciente } \\
\text { oncológico. }\end{array}$ & $\begin{array}{l}\text { II - Esfera de influência } \\
\text { paciente/cliente }\end{array}$ \\
\hline A9 - Malloy P., et al. & $\begin{array}{l}\text { O estudo apresentou o câncer como terceira maior causa de morte no Quênia e } \\
\text { que os enfermeiros são os principais cuidadores, avaliando e gerenciando esses } \\
\text { pacientes na clínica, em ambientes de internação e em comunidades rurais e } \\
\text { remotas. Informa que para a Organização Mundial de Saúde os enfermeiros } \\
\text { que prestam estes cuidados no Quênia estão empenhando esforços para apoiar } \\
\text { as necessidades contínuas dos doentes a fim de promover cuidados paliativos e } \\
\text { prevenir seu sofrimento. }\end{array}$ & $\begin{array}{l}\text { III - Esfera Organização de } \\
\text { Sistemas }\end{array}$ \\
\hline
\end{tabular}

Fonte: Autores (2018).

\section{Discussão}

Esta revisão integrativa que diz respeito aos cuidados realizados por enfermeiros não especialistas ao cliente oncológico hospitalizado apresentando em destaque um primeiro grupo de estudos constituído por: A1 - descreve que enfermeiros realizam orientações e intervenções no tratamento quimioterápico, A2 - o enfermeiro faz avaliação da lesão, escolha da cobertura e curativos de feridas tumorais, A3 -o enfermeiro tem como competência realizar estratégias para controle da situação do paciente intervindo no momento necessário para evitar complicações, minimizando ou promovendo melhora dos efeitos adversos do tratamento ao paciente submetido a altas doses de quimioterápicos, A6 - afirma que o enfermeiro, junto à criança com câncer em cuidados paliativos, dá conforto, cuida da família, atende às necessidades gerais da criança, proporciona qualidade de vida,; dá apoio espiritual, emocional e religioso e, A7 - enfermeiros desempenham atividades que englobam o cuidado espiritual à pessoa com câncer. Este primeiro grupo constituído por cinco estudos é o maior formado e possibilita uma correspondência das ações dos enfermeiros não especialistas em oncologia com as competências básicas da Esfera de influência do Enfermeiro na prática da enfermagem conforme a ONS.

Neste âmbito,o Enfermeiro Oncológico Clínico Especialista avança as práticas de enfermagem e melhora os pacientes sensíveis a suas intervenções, atualizando e melhorando normas e padrões da prática de enfermagem oncológica, fornecendo liderança, conhecimento, habilidades e novos modelos de comportamento que influenciam no próprio cuidado e também no desenvolvimento de políticas, procedimentos e protocolos baseados em evidência, bem como nos modelos e diretrizes para melhores práticas (ONS, 2008).

É também possível correlação com o modelo de competências descrito por Zarifian como competência técnica, no qual os profissionais devem ter conhecimentos específicos sobre o trabalho a ser realizado (Zarifian, 1999).

Um segundo grupo identificado neste estudo é constituído pelo A4, no qual descreve que o enfermeiro ressignifica a gerência da interação com os homens com câncer em tratamento antineoplásico, a fim de assegurar a integralidade do cuidado mediante a perspectiva de gênero; pelo A5 que apresenta a elaboração do cuidado em um subconjunto terminológico realizado por enfermeiros, ressaltando a importância destes profissionais diante de um cuidado baseado em evidências científicas, para uma ação de enfermagem segura no manejo dos sinais e sintomas das feridas tumorais e também em dados para o ensino e 
pesquisa; e pelo A8 que ressalta mudanças que podem ser realizadas por enfermeiros, com o objetivo de qualificar a assistência e contribuir para minimizar a dor destacando: a implantação da dor como quinto sinal vital, formação de grupos de estudo e a realização de pesquisas com enfoque na mensuração da dor e na assistência ao paciente oncológico. Esse grupo formado por 03 artigos possibilita uma correspondência das ações realizadas pelo Enfermeiro não especialista com a Esfera de influência paciente/cliente dos Enfermeiros Oncológicos Clínicos Especialista proposta pela ONS.

Esta descreve que o Enfermeiro Oncológico Clínico Especialista usa conhecimentos e habilidades para avaliar, diagnosticar, manejar a doença e comportamento de risco nos pacientes com diagnóstico pregresso, atual e potencial de câncer, demonstrando também fundamento e competência para a realização e avaliação de intervenções inovadoras, custo-efetivas de qualidade para solucionar problemas da doença, a fim de melhorar resultados junto aos pacientes atendidos (ONS, 2008).

Desta forma, é possível também uma correlação com o modelo descrito por Zarifian (1999) como competências sociais no qual o profissional deve saber ser, incluindo o desenvolvimento de atitudes que sustentam os comportamentos das pessoas.

O estudo A9 trata sobre enfermeiros não especialistas do Quênia que empenham esforços para apoiar as necessidades contínuas dos doentes de câncer de modo a promover cuidados, buscando prevenir o sofrimento na situação de pobreza possibilitando uma correspondência com a Esfera da organização dos sistemas proposta pela ONS.

Esta esfera aponta que o Enfermeiro Oncológico Clínico Especialista influencia organizações e sistemas de assistência, articulando contribuições de cuidados de enfermagem oncológica como defensor da enfermagem profissional para melhorar os pacientes com câncer. Fornece liderança à grupos de enfermagem e interprofissionais a fim de organizar programas de assistência inovadores, que atendem às necessidades dos pacientes em uma linha de cuidado completa, provê conhecimentos e habilidades para estimular alterações nos sistemas que promovem melhorias práticas de enfermagem oncológica, de qualidade e resultados custo-eficazes (ONS, 2008).

Assim, é possível correlação ao modelo competência sobre processos de trabalho descrito por Zarifian que informa a necessidade do conhecimento e influência no processo pelo profissional (Zarifian, 1999).

Portanto, segundo a pesquisa, a exemplo de Enfermeiros Oncológico Clínico Especialista, o enfermeiro não especialista realiza diversos cuidados ao cliente oncológico hospitalizado, no entanto, necessitam estar fundamentados no conhecimento técnico-científico, uma vez que na abordagem em oncologia é necessário um preparo para qualquer tipo de resultado, quando se tem grandes chances de cura, um prognóstico fora da possibilidade de cura ou sequelas em qualquer escala. Neste contexto, o paciente em todo o percurso da doença necessita de cuidados e uma equipe preparada, pois apresenta muitas fragilidades, sendo elas tanto físicas como psicológicas, principalmente quando seu diagnóstico não possibilita mais terapêutica de cura (Coelho, 2017).

Observa-se que alguns artigos deste estudo sinalizaram a existência de fragilidades na assistência frente ao cuidado generalista, caracterizando uma maior necessidade de conhecimento da oncologia pelos enfermeiros não especializados, principalmente frente aos índices epidemiológicos do câncer na atualidade (Lucas, 2020).

Nesse contexto, considerando a complexidade desse cuidado, a identificação e a compreensão das estratégias de enfrentamento do câncer podem servir ao planejamento curricular da formação de futuros enfermeiros, contribuindo para melhor qualificar a assistência de enfermagem. Devendo ser capaz de suprir lacunas existentes na preparação deste profissional para lidar com a adversidade típica da enfermagem oncológica (Santos et al., 2016).

Faz-se necessário a reflexão sobre o atual perfil epidemiológico das neoplasias e, quanto ao seu aumento expressivo para exigir um maior nível de conhecimento dos enfermeiros que terão contato cada vez maior com o cliente oncológico nos diversos níveis de atenção à saúde no âmbito do Sistema Único de Saúde (SUS). Portanto, justifica-se a indispensabilidade de 
capacitações e educação continuada permanente desses profissionais, desde os que atuam em unidades hospitalares exclusivamente oncológicas, até os que trabalham em outras áreas e estratégias da saúde (Cruz \& Rossato, 2015).

Neste cenário, é fundamental aos enfermeiros perceber e apreender que é de sua responsabilidade a assistência segura e livre de danos aos pacientes e a utilização de ferramentas como protocolos, planos terapêuticos, notificação de eventos adversos e planos de ação para ampliar e melhorar a práxis profissional, alicerçada nas políticas de qualidade e nos princípios éticos da profissão (Silva et al., 2018).

Desta forma, apesar do atual cenário da saúde com tecnologias cada vez mais modernas, as múltiplas atividades laborais do enfermeiro, além da exacerbação do capitalismo, exigem a necessidade de um cuidado em enfermagem ético e humano, baseado em habilidades, competências, atitude profissional e valorização da subjetividade de cada ser cuidado. Os enfermeiros, por terem o cuidado como essência do ser e da própria profissão, precisam cuidar com uma atitude de ocupação e preocupação, unindo a competência técnica com a sensibilidade, afetividade e o respeito (Santos et al., 2017).

À vista disso, a Educação Permanente em Saúde (EPS) é uma estratégia político-pedagógica que toma como objeto os problemas e necessidades emanadas do processo de trabalho em saúde, e relaciona o ensino, a atenção à saúde, a gestão do sistema e a participação do controle social. Objetivando qualificação e aperfeiçoamento do processo de trabalho em vários níveis do sistema, orientando-se à melhoria do acesso, qualidade e humanização na prestação de serviços e para o fortalecimento dos processos de gestão político-institucional do SUS no âmbito federal, estadual, municipal e local (Ministério da Saúde, 2018).

Considera-se também que, além da especialização, a Educação Permanente em Saúde desempenha um importante papel no contexto da assistência, já que se relaciona diretamente com a própria qualidade da assistência, autonomia profissional, integralidade e ética social, sendo de responsabilidade das instituições de ensino, inclusive preconizada pelas diretrizes curriculares nacionais, a formação de profissionais competentes para se educarem permanentemente em saúde (Lima \& Ribeiro, 2019).

\section{Limitações do estudo}

Como limitação do estudo aponta-se o número reduzido de artigos (nove artigos científicos), selecionados pelos critérios de inclusão previamente estabelecidos e descritos na metodologia e também o nível de evidência relacionado aos artigos encontrados. Pode-se perceber a incipiência dos estudos científicos publicados caracterizando que se trata de temática ainda em desenvolvimento.

\section{Contribuições para a enfermagem}

A principal contribuição deste estudo é o estímulo ao pensamento crítico relacionado ao cuidado realizado pelo enfermeiro não especialista em oncologia no atual contexto da assistência oncológica no Brasil e no mundo. Ressalta-se que suas ações vão além das tradicionais da profissão, sendo estes, cada vez mais responsáveis por funções mais complexas, resultantes dos avanços dos métodos de diagnóstico e tratamento prestado a essa clientela. Portanto, ao oportunizar ao leitor uma reflexão crítica sobre a realidade, esta pesquisa fornece subsídios que podem contribuir no planejamento curricular da formação dos enfermeiros e em projetos de Educação Permanente com vistas à melhor promoção da assistência ao cliente oncológico, a comunidade na qual está inserido e a sociedade como um todo.

\section{Conclusão}

O estudo apontou que enfermeiros não especialistas em oncologia mobilizam diversas competências ao cuidar do 
cliente oncológico hospitalizado utilizando conhecimentos e habilidades gerais em seu cotidiano de trabalho, no entanto, desconhecem seu protagonismo como agente de mudança frente à epidemiologia do câncer. Neste contexto, diante da necessidade de se repensar a formação curricular, como medida urgente a Educação Permanente por ser ferramenta potente para uma nova postura dos profissionais frente à realidade apresentada. Assim, os enfermeiros precisam vencer as diferentes barreiras na utilização de resultados de pesquisas na prática profissional a fim de superar possíveis lacunas no conhecimento, principalmente considerando a complexidade relacionada com a assistência oncológica. Ressalta-se a necessidade de mais pesquisas e estudos sobre o assunto a fim de fomentar novas evidências científicas e contribuir para melhores resultados sobre a temática.

\section{Referências}

Barp, M., Carneiro, V. S. M., Amaral, K. V. A., Pagotto, V., \& Malaquias, S. G. (2018). Cuidados de Enfermagem na prevenção do tromboembolismo venoso: revisão integrativa. Revista Eletrônica de Enfermagem, 20 (14). 10.5216/ree.v20.48735

Cardoso, V., Trevisan, I., Cicolella, D. A., \& Waterkemper, R. (2019). Systematic Review of mixed methods: method of research for the incorporation of evidence in nursing. Texto contexto - Enferm, 28.https://doi.org/10.1590/1980-265x-tce-2017-0279

Cruz, FS., Rossato, L. G. (2015). Cuidados com o Paciente Oncológico em Tratamento Quimioterápico: o Conhecimento dos Enfermeiros da Estratégia Saúde da Família. Revista Brasileira de Cancerologia, 61 (4). Recuperado de http://www1.inca.gov.br/rbc/n_61/v04/pdf/04-artigo-cuidados-com-o-pacienteoncologico-em-tratamento-quimioterapico-o-conhecimento-dos-enfermeiros-da-estrategia-saude-da-familia.pdf

Coelho, J. P. S. L. (2017). Assistência de enfermagem frente ao paciente oncológico. Revista Gestão Universitária, 7, 1-10. http://www.gestaouniversitaria.com.br/artigos-cientificos/assistencia-de-enfermagem-frente-ao-paciente-oncologico.

Gadelha, M. I. P. (2018). 30 years of Cancer Care in the Brazilian National Health System. Revista Brasileira de Cancerologia, 64(2). https://doi.org/10.32635/2176-9745.RBC.2018v64n2.83

Instituto Nacional de Câncer José Alencar Gomes da Silva. (2019). Estimativa/2020: Incidência de Câncer no Brasil. Rio de Janeiro: INCA. Recuperado de https://www.inca.gov.br/sites/ufu.sti.inca.local/files//media/document//estimativa-2020-incidencia-de-cancer-no-brasil.pdf

Lima, L. P. S. \& Ribeiro, M. R. R. (2019). A competência para Educação Permanente em Saúde: percepções de coordenadores de graduações da saúde. Physis Rev de Sal Col online, 26 (2). https://doi.org/10.1590/S0103-73312016000200008

Lins, F. G. \& Souza, S. R. (2018). Formação dos enfermeiros para o cuidado em oncologia. Rev. enferm. UFPE online, 12 (1). https://doi.org/10.5205/19818963-v12i01a22652p66-74-2018

Lucas, D. M. S. (2020). A competência do enfermeiro não especialista em oncologia no cotidiano do cuidado hospitalar. [Dissertação do mestrado, Universidade Federal do Estado do Rio de Janeiro].

Mendes, K. D. S.; Silveira, R. C. C. P., \& Galvão, C. M. (2008). Revisão integrativa: método de pesquisa para a incorporação de evidências na saúde e na enfermagem. Texto Contexto Enferm, 17 (4). https://doi.org/10.1590/S0104-07072008000400018

Moura, D. C. A. de, Greco, R. M., \& Leonel, M. (2016). Saúde Do Trabalhador - Produção Científica Da Enfermagem Na Primeira Década Do Século XXI. Revista De Enfermagem Da UFJF, 1(2). https://periodicos.ufjf.br/index.php/enfermagem/article/view/3800

Oncology Nursing Society (2008). Competências do Enfermeiro Oncológico Clínico Especialista. https://www.ons.org/sites/default/files/cnscomps.pdf

Polit, D. F., \& Beck, C. T. (2018). Fundamentos de pesquisa em enfermagem: avaliação de evidências para a prática da enfermagem. (9a ed) Artmed.

Paiva, C. J. K. de, \& Cesse, E. Ângela P. (2015). Aspectos Relacionados ao Atraso no Diagnóstico e Tratamento do Câncer de Mama em uma Unidade Hospitalar de Pernambuco. Revista Brasileira De Cancerologia, 61(1), 23-30. https://doi.org/10.32635/2176-9745.RBC.2015v61n1.374

Conselho Nacional de Saúde. (2016). Resolução no510/16. http://conselho.saude.gov.br/resolucoes/2016/Reso510.pdf

Santos, A. G., Monteiro, C. F. S., Nunes, B. M. V. T., Benício, C. D. A. V., \& Nogueira, L. T. (2017) O cuidado em enfermagem analisado segundo a essência do cuidado de Martin Heidegger. Rev Cubana Enferm. 33(3). http://www.revenfermeria.sld.cu/index.php/enf/article/view/1529

Santos, N. A. R., Santos, A. T. C., \& Silva, R. P. (2016). Coping strategies of nurses in the care of patients with head and neck neoplasms. Rev Esc Enferm USP. 50 (4). doi.org/10.1590/S0080-623420160000500005

Ministério da Saúde (2018). Planejamento das Ações de Educação Permanente em Saúde no Sistema Único de Saúde: Orientações / Ministério da Saúde (1 ed.). Secretaria de Gestão do Trabalho e da Educação na Saúde, Departamento de Gestão da Educação na Saúde. https://portalarquivos2.saude.gov.br/images/pdf/2018/marco/28/Manual-Tecnico-PRO-EPS-SUS-MINUTA17-10.pdf

Silva, A. $\mathrm{T}$ et al. (2018). Segurança do paciente e a atuação do enfermeiro em hospital. Rev enferm UFPE on line. 12(6). https://periodicos.ufpe.br/revistas/revistaenfermagem/article/viewFile/234593/29174 
Research, Society and Development, v. 10, n. 6, e2910615306, 2021

(CC BY 4.0) | ISSN 2525-3409 | DOI: http://dx.doi.org/10.33448/rsd-v10i6.15306

Silva, F. C.,Cunha, C. S.,Feitosa., T. S. R. G. T., Silva, A. D. M. \& Sousa, I. D. B.Assistência de enfermagem a pacientes com câncer em cuidados paliativos: revisão integrativa. Revista Enfermagem Atual, 90(21). https://revistaenfermagematual.com.br/index.php/revista/article/view/626/632

Stillwell, S. B., Fineout-Overholt, E., Melnyk, B. M., \& Williamson, K. M. (2010). Searching for the Evidence: Strategies to help you conduct a successful search. American Journal of Nursing. 110 (1). https://pubmed.ncbi.nlm.nih.gov/20520115/

Zarifian, P. (1999.) Objectif compétence. Liaisons. https://revistas.pucsp.br/psicorevista/article/viewFile/16656/12510. 